\title{
FROM PHYSICAL TO EMOTIONAL PAIN IN CHRONIC KIDNEY DISEASE: NURSES' PERCEPTIONS
}

\author{
Guillermo Pedreira Robles ${ }^{1}$, Mariela Patricia Aguayo González ${ }^{2}$. \\ ${ }^{1}$ MSN, BScN, RN. Nephrology Department. Parc de Salut Mar. Barcelona. Spain. \\ ${ }^{2} \mathrm{PhD}, \mathrm{MSN}, \mathrm{BScN}, \mathrm{RN}$. Nursing Department. Faculty of Medicine. Universitat \\ Autònoma de Barcelona (UAB). Spain.
}

CORRESPONDENCE:

Guillermo Pedreira-Robles

Nephrology Department. Parc de Salut Mar. Barcelona. Spain.

Passeig Maritim 25-29. 08003. Barcelona. Spain,

Email: gpedreira@parcdesalutmar.cat

\section{DECLARATIONS}

Financing: This project was awarded with the Jandry Lorenzo Scholarship of the Spanish Nephrology Nurses Association (SEDEN) endowed with $€ 1,800$, which were devoted to financing the data collection and analysis.

Conflict of interests: None.

\section{Author contributions}

GPR: Principal investigator, participated in the design of the study, supervised data collection and analysis, helped draft manuscript, approved the final manuscript.

MPAG: Participated in the design of the study, participated in data analysis, helped draft manuscript, approved the final version.

\section{Acknowledgment}

To Gail Craigie for her support in the translation process and to the Hospital del Mar nephrology nurses for sharing their perceptions. 


\section{SUMMARY}

Background: Changes in body image are a recurring theme in the literature on CKD. There is insufficient evidence identifying nurses' perceptions of this topic.

Objective: To explore nurses' perceptions of changes in the bodies of persons with CKD.

Design: Phenomenological study.

Methods: Data were collected by semi-structured interviews with nephrology nurses selected through pragmatic sampling until data saturation. The interviews were transcribed and analyzed using the 7 phases of Colazzi.

Findings: Information saturation was achieved with 6 nurses. Coexisting elements were found between the physical and emotional impact of CKD and therefore the data were organized into a central theme: 'From physical to emotional pain in the individual's body'. This central theme was defined by 7 subthemes that describe the coexistence of these two physical and emotional spheres, giving rise to the appearance of important meanings in the changes occurring in the bodies of individuals diagnosed with CKD. These meanings included stigma, dysfunctionality, emotional pain, the feeling of slavery, lack of information, taboo and hope.

Conclusions: In the nurses' perspective, the changes occurring in the bodies of people with CKD can transcend the physical sphere and acquire more complex meanings. Nurses have unique insight into these complex meanings, which include all the domains of the person, and which have important implications for daily practice.

Implications for practice: Nurses should have specialized training in providing effective responses to patients' emotional needs. It is also important to incorporate professional profiles that support nursing interventions.

Keywords: kidney disease, emotional state, body perception, health education. 


\section{BACKGROUND}

Chronic kidney disease (CKD) is characterized by an irreversible loss of renal function for more than 3 months. According to the "Catalan Register of Renal Patients" (Martínez-Castelao, et al., 2017), the prevalence and incidence rates of this disease continue to grow, while mortality has remained stable at around $8 \%$ for the last 8 years. This indicates that the prevalence of $\mathrm{CKD}$ is progressively rising due to longer life expectancy and the increasing presence of other associated diseases such as diabetes, high blood pressure, and heart disease. In Spain, around $10 \%$ of the general adult population (> 30 years) has CKD (Vargas-Marcos, 2015).

Because of the loss of renal function, persons diagnosed with CKD will usually undergo one of the available renal replacement techniques: haemodialysis, peritoneal dialysis, or kidney transplant (Pendse, Singh and Zawada Jr, 2006). Patients usually follow a single treatment modality but, at some point in their lives, they may require a combination of up to all 3 modalities, leading to the presence of several scars on their bodies (Sadeghian, Masroor-Roudsari, Seyedfatemi and Rafiei, 2016). In addition, individuals experience other physical effects due to drug therapies and the surgical or replacement techniques they may require, depending on their needs (Silva, et al., 2018), which affect all dimensions of their lives.

Therefore, CKD strongly impacts people's lives after diagnosis (Zalai, Szeifert and Novak, 2012). The process of the disease and its progression favour the appearance of limitations, frustrations, and biopsychosocial changes affecting individuals' quality of life. Recurrent themes in the literature on kidney disease include changes in body image, altered sleep patterns, weight loss or gain, loss of sexual interest, professional difficulties, and changes in family relationships and roles (Malheiro-Oliveira and Arruda-Soares, 2012).

In this regard, a review by Ormandy (2008) highlighted physical symptoms and body image as being among the most important elements in CKD and in the personal satisfaction of affected individuals with the information process. Likewise, several studies have reported that individuals experience a loss of control over their bodies, impaired quality of life as a result of changes in physical appearance, and acceptance of the need for dialysis but rejection of its effects on the body; in addition, people with 
CKD report body changes with all renal replacement modalities, with no treatment producing more physical effects than others, as well as psychological alterations due to changes in body image, etc. (Lewis and Arber, 2015; Malheiro-Oliveira, et al., 2012; Sadeghian, et al., 2016; Silva, et al., 2018; Partridge, et al., 2011).

This type of patient will attend nephrology services for a long time, where nursing interventions will be decisive in acceptance of the disease and in teaching self-care. These elements are included within some nursing knowledge models. Callista Roy's adaptation model (Roy, Whetsell and Frederickson, 2009) views self-image as a determining area in constant adaptation with the environment that, at the same time, is also constantly changing. In the nursing assessment theory proposed by Marjory Gordon (Gordon, 1994), self-image and self-perception are also considered key elements in nursing assessment. Currently, this assessment model is still the basis for care planning in most centers in Spain.

Despite these theoretical models and evidence of the importance of these concepts in the above-mentioned studies, there are no systematic reviews on the alterations or limitations caused by CKD in the body image of affected individuals. Moreover, an important gap is the lack of studies on nurses' perceptions of these topics. Nurses are acknowledged as a key figure in person-centered care, but there are no studies in the literature on their role in caring for people with CKD that focus on body image (Partridge and Robertson, 2011). Consequently, identifying nurses' perceptions on the subject is of particular interest. These perceptions could influence the basis of care provided in daily practice. It is also important to identify any discrepancies to address the problem from a perspective common to all the parties involved.

Therefore, the objective of this study was to explore nurses' perceptions of the changes occurring in the bodies of persons with CKD throughout their disease process. 


\section{MATERIAL AND METHODS}

\section{DESIGN}

We conducted a qualitative study, based on the phenomenology approach (Heidegger, 2010), with the main aim of examining the nature and meanings of the phenomenon to be studied: nurses' perceptions of the changes occurring in the bodies of people with CKD.

\section{PARTICIPANTS}

The participants in this study were 6 nurses from a nephrology service in a university hospital of Barcelona. Participant selection was governed by the principles of pragmatic sampling until data saturation was reached, indicated by the lack of new themes present in the data (Kuper, Lingard and Levinson, 2008). Thus, data saturation was achieved after assessment of each interview, including repetition of ideas, the depth of the concepts and the appearance of new topics. Saturation was achieved when no new information was provided by the informants.

The use of pragmatic sampling in a closed nephrology service in which professionals share the same daily work, clinical sessions, reflective spaces, training opportunities, etc., allowed data saturation to be achieved with a small sample, revealing shared knowledge and joint reflection. Nevertheless, data were collected and analyzed rigorously.

The participating nurses were contacted in person in their workplace and were informed of the present study. This procedure facilitated quick and easy contact with the informants. This was helped by the fact that the principal investigator worked with them. After this phase, we assessed the inclusion and exclusion criteria. Finally, we included 6 nephrology nurses with more than 5 years' experience. We excluded nurses who themselves had kidney disease or any other personal relationship with the disease, since their inclusion could lead to a bias in the information to be analyzed. The main relevant data of the participants are shown in Table 1. 


\section{RESEARCH TEAM POSITIONS}

According to the bracketing strategies proposed by Chan, et al. (2013) we used reflexivity as a key point throughout the research process to help us identify our potential influence on the process. Reflexivity involves conducting an honest examination of the values and interests of the researchers that impinge upon research work in all its phases. Thus, bringing reflexivity into consciousness allowed us to be aware of possible biases throughout the study process.

An important point is that the principal investigator of this study works in the same nephrology service as the selected informants. Before initiating the data collection and after the literature review, the initial presuppositions were defined and those ideas that could interfere with the results were identified. Thus, we confirmed that we put aside all our own assumptions and adopted the proper attitude to guarantee the rigor of the research, using the four bracketing strategies proposed by Chan, et al. (2013): the strategy for mental preparation, confirming that we set aside all our previous knowledge and maintained that attitude throughout the research; the strategy for deciding the scope of the literature review, confirming that the knowledge gained did not affect our preconceptions on the topic under study; the strategy for planning data collection, ensuring that the semi-structured interview used was characterized by open-ended questions that were developed in advance and by trained staff and the strategy for planning data analysis, confirming that the researchers had suppressed their preconceptions during data analysis and validating it by returning the analysis to the study participants using Colaizzi’s data analysis method described above.

Although it might have been appropriate for the interview leader to have no direct relationship with the informants, we preferred to maintain this relationship because having an interviewer with a shared professional experience may result in richer data. At the same time, the presence of an external investigator within the research team, who was non-working in the same area and was not directly connected with the informants, allowed us to maintain this distance between personal ideas and the analysis.

\section{DATA COLLECTION}

Information was collected through semi-structured face-to-face interviews, carried out in a closed office in the workplace. The participants had the opportunity to choose 
the space (outside or inside the work center) where they expected to feel most comfortable for the interview. The interviews were conducted between April and May 2018 by the main author of the study who also explained it to the participants and collected the informed consent forms. In these interviews, open questions were asked, based on the literature review conducted prior to the study, aiming to meet the objectives of the study (Appendix 1). The interviews lasted between 40 and 60 minutes, approximately, and were recorded after the participants provided their authorization and informed consent. The interviewer collected field notes to highlight important aspects of the interview, which were important at the time of the analysis.

\section{ANALYSIS}

Colaizzi's 7 stage process (1978) was used for the analysis. This process provides a rigorous analysis and is closely based on the data. The result is a concise description of the phenomenon under study, which is validated by the participants. In the first phase of this process, the interviews were read to acquire a sense of their meaning as a whole; then, significant statements were extracted and meanings were formulated based on the significant statements; next, these meanings were categorized into conglomerates of themes to identify common experiences among the informants; these results were then incorporated into a rich and exhaustive description of the experiences analyzed. This process was carried out manually without the use of computer support software. In the second phase, the result of the analysis was given to the informants for validation (data triangulation) but no new contribution was incorporated into the discourse, thus validating the contributions already made by the participants. Themes were defined through consensus among the members of the research team, who held regular meetings (after each interview) to discuss the results, thus achieving data triangulation. Data saturation was also discussed by the research team members.

The process described is equivalent to maintaining the credibility, transferability, dependence and confirmability criteria in the data collection and analysis process described in the literature (Guba, 1981). Concerning credibility, we attempted to ensure that the relevant nurses' perceptions captured had the same isomorphic structure recognized by the study informants. Regarding transferability, we also attempted to establish new and important knowledge about a specific topic and, at the same time, that allowed us to transfer the same conclusions to similar contexts, such as: other 
nephrology nurses, other nephrology services and other persons with CKD. To achieve data consistency and avoid instability in the information that was collected and analyzed, concerning dependence, specific procedures were used, such as triangulation of researchers and results and the constant comparison strategy to review and compare the emerging results with previously formulated theories. Finally, data triangulation and the development of a self-critical conscience on the part of the principal investigator guaranteed neutrality in data collection and analysis, guaranteeing confirmability. This refers to the maintenance of self-critical awareness during the four bracketing strategies proposed by Chan, et al. (2013), mentioned above.

\section{ETHICAL CONSIDERATIONS}

This study was conducted in accordance with the basic principles guiding healthcare and research in the field of social health. Data confidentiality was guaranteed in accordance with the European Regulation 2016/679 on Data Protection and the Organic Law on Data Protection and Guarantee of Digital Rights of Spain. Before data collection, all participants provided written informed consent. This project was approved by the Research Ethics Committee of the hospital where the study was performed (number 2018/7889/I). 


\section{FINDINGS}

A central theme and 7 subthemes emerged from the data (Table 2). The nurses interviewed believed there was a close relationship between the physical and emotional impact of CKD, as they identified elements intervening and coexisting in people's bodies. This general premise led to the emergence of the central theme of the study:

\section{From physical to emotional pain in the body of persons with CKD.}

This central theme acquired its full meaning from the subthemes comprising it: the stigma created by the need for dialysis, the dysfunctionality accompanied by pain, the feeling of slavery, lack of patient information, the silence of taboo, lack of resources and hope for improvement. The nurses identified these obvious problems resulting from kidney disease and the interventions and treatments followed by people with this disease. These treatments and physical interventions also affected the emotions and psyches of people with CKD. The impact of these problems varied depending on the complications that occurred, but, in the main, the nurses depicted CKD and its treatment as leading to emotional and physical pain. All this emotional impact, as well as the care of persons with $\mathrm{CKD}$, necessitates a complex approach that is not limited to the physical treatment of the disease. This complexity, which is described below through the analysis of the subthemes, involves both physical and emotional components.

\section{STIGMA CREATED BY THE NEED FOR DIALYSIS}

Nurses reported that persons with CKD were differentiated from healthy persons by their permanent dialysis access. They identified this access as a physical mark, an aesthetic scar, a mechanical device that was visible and notably marked the body, giving rise to the subtheme of stigma created by a need.

N1: In the bodies of people with kidney disease there will be either a fistula or a catheter or a catheter for peritoneal dialysis. All this leads to an obvious change in their bodies. Some people cope with it better than others, especially the fistulas that make people ask them about it more, since they are very large and quite obvious. For example, in summer, when wearing short sleeves, many people tell you that it's a problem. Then we have the individuals with peritoneal access who, in their intimate lives, can have problems. 
The emotional impact of dialysis access identified by nurses, beyond appearance, were connected to the concept of the body as an element of social relationship with people and the environment. This relationship may be adversely affected in persons with dialysis access.

N2: If the fistula becomes bigger or more obvious, it can have certain implications for the patient. We know that a fistula, when you touch it, has a characteristic thrill, and so... it is still a strange sensation. People ask them about this strangeness. So, both physically and mentally, it can have an effect. You relate to others and it provokes a certain rejection. The body is the one thing that brings you closer to the other and, in some way, these characteristics can be an impediment. In fact, some people, who have received a transplant, have even asked for the fistula to be removed, because, in some way, it is like transforming the body. Like it spoils it.

\section{DYSFUNCTIONALITY ACCOMPANIED BY PAIN}

Beyond the aesthetic component or the effects of dialysis access identified by nurses in persons with $\mathrm{CKD}$, the functionality of the access led to more evident involvement as described by the nurses themselves. A dysfunctional access, hampering access and causing pain, was one of the most negative aspects perceived by persons with CKD according to the nurses treating those developing these complications.

A dysfunctional access caused both physical pain and emotional pain. People with CKD found repeated cannulation of the access physically painful and emotionally distressing and would dread the procedure, especially as they did not know who would perform it or how long they would have to wait.

Nurses reported that people with access dysfunction thought constantly about it and the treatment. Thus, the pain or physical complication was accompanied by emotional pain outside treatment hours and was constant in people's lives.

N3: I believe that as in everything, a fistula, or an access, complicated, that is difficult to cannulate, that if I do not find the professional that knows about it... I will be here longer, or bruises will come out, or they will insert the needle more. It is very strong the distress, the anxiety that this generates. The fact of coming to dialysis every two days and thinking that you will find all this... I think it is very negative for the person who comes. It is no longer the simple fact of "it hurts me". I mean: needling with these thick needles hurts. But everything that surrounds this process, the worry that comes from the uncertainty, who will I see? Will she do it well or won't she do it well? Will the dialysis be longer because maybe we will be there an hour doing and undoing? This is very burdensome for me. 


\section{THE FEELING OF SLAVERY}

Due to the above-mentioned difficulties that can develop after placement of dialysis access and, consequently, with treatment, another of the key elements identified by nurses was the feeling of slavery among people with CKD. The nurses interviewed linked the physical aspect of the treatment with its emotional impact on the patient, concluding that these treatment modalities can be perceived as a necessary slavery to stay alive.

Renal replacement therapies, through either of the two existing dialysis modalities, require a heavy time investment. Because persons with CKD must invest this time in a medical treatment that may lead to complications and be unpleasant, they may have to sacrifice other, more rewarding activities. The constant repetition of treatment (either every day in the case of peritoneal dialysis or every two days in the case of haemodialysis) and the knowledge that it could last for the rest of their lives can, according to the nurses' perceptions, lead affected individuals to have a feeling of slavery.

N3: What affects the person very badly is this process of slavery and being connected four hours a day, every two days. Not being able to lead a normal life, to spend hours recovering, to be well again ... all this is what I think is the most horrible thing about this disease.

\section{LACK OF PATIENT INFORMATION}

The nurses believed that persons with CKD were not well informed of treatment complications (such as having very dry skin or itching), or of their emotional impact (such as sadness or anxiety). They reported that people with CKD were not informed of certain aspects related to the disease and its treatment and, consequently, that they failed to understand issues that were important for their emotional well-being. This lack of information was related to the two following subthemes: the silence of taboo and the lack of resources. In addition, the nurses acknowledged that, on occasion and for various reasons that will also be discussed below, they focused more on technical aspects or the physical side of the treatment and its complications, neglecting other elements that are equally important for people's well-being, such as body image.

N5: I am not sure that people with CKD realize that the symptoms of the disease can be due to the treatment or dialysis. Or if they really know that what happens to them is related to some type of medication or treatment. They simply complain about how they feel. There are some 
things that are very clear such as, for example, in the case of vascular access. They need a fistula or a catheter to have dialysis and the possible effect of the treatment is more direct. But there are also other symptoms that don't have such a clear a cause-effect, as is the case, for example, of the skin. Having very dry skin or itching is less often attributed by individuals to the treatment. Maybe they are not aware, and nobody has explained it to them. This is wrong because we should explain it to them clearly.

Despite the gaps in patient information, two other equally important elements were directly related to the lack of understanding, namely: the silence of taboo and the lack of resources, which are discussed below.

\section{THE SILENCE OF TABOO}

Nurses reported that there was a taboo on discussing sexual problems, the effects of CKD in young people, treatment impact, and the implications for social life, physical appearance and self-esteem, etc.

N6: I don't talk about sexuality with people with CKD. It's a taboo subject. I think it's uncomfortable for them and for us. Most of the patients we have are old people and we understand that they don't have sex.

Nurses identified this taboo clearly in their discussion of sexuality and other bodily and emotional impact. Although young people do not constitute the majority of patients with CKD nor are they the most representative group, they were identified by nurses as being those most clearly affected in these areas.

$\mathrm{N} 2$ : The younger the patient is, the feeling is that, in their relationships, because this can condition or may cause, especially with the other person with whom they are going to have a relationship, a discomfort. Therefore, it is very important to talk openly about these issues with them without there being any taboos. Sometimes it is the people with CKD themselves who put up this barrier, but at other times it is us.

\section{LACK OF RESOURCES}

Apart from the consequences of the taboo, nurses felt that they lacked enough resources to address psychological issues related to the impact of CKD on people's bodies.

N1: There are key people or professionals who should intervene beyond what nurses do. There are things that we cannot get to as nurses. All that we have said and the emotional effects of the physical disease should be guided by a psychologist. The training that I, as a nurse, have received, is limited and the support process in relation to limitations, frustrations, effects, etc., should be carried out by a qualified professional. Moreover, this psychologist should also train us to be able to do what we are already doing in a more professional way. Sometimes, one's selfperception can't be expressed by words alone. If all the spheres of your life are involved in a vital change, this requires extreme sensitivity. 
Throughout the disease process, people with CKD will experience various situations that may lead them to feel major losses related to their body image. In the Spanish publicly-funded health system, these losses are not formally identified and supported. As a result, nurses (who assume this part of the care) sometimes feel that they do not have control of the situation. On the other hand, they reported that they did intervene in these needs using the strategies learned in daily clinical practice over years of experience of treating people with CKD. However, the discourse analyzed revealed that the nurses felt that they did not have all the necessary skills and resources necessary to address their patients' emotions, mourning, losses, etc., possibly because it is not a direct nursing competence or because of a lack of training in the subject or the heavy workload.

N3: One thing that is not done, sometimes, not offering or not taking care as it should be, is the lack of time. Besides, a strategy that would be good to add is training.

N4: Nurses play a very important role in the emotional support of patients with chronic kidney disease, although sometimes we do not go so deep. But we should, and we should give it the importance it deserves.

This lack of resources, knowledge, time, improvement strategies, training, etc., described by the nurses and that hindered their ability to provide emotional support to people with CKD may lead them to exclude these aspects and to carry out patient care in a mechanical way. The nurses believed that they were currently doing good work but that more resources would allow them to provide greater support to people with CKD.

\begin{abstract}
N4: We should give it it's due importance and, usually, we don't. It's true, and now, when I say it, it makes me think a lot. Everything else is very important because life depends on that. And the other part is only, between very large quotation marks, the physical aspect and the psychic repercussions that they may have. It doesn't kill you as much as eating a kilo of cherries. Then, it is further back or more apart. It remains in the background and we focus on the immediacy of the most "important" problems.
\end{abstract}

\title{
HOPE FOR IMPROVEMENT
}

Finally, another key element in the nurses' discourse was hope for improvement. While the scars caused by dialysis access and dysfunction involved negative experiences for nurses, the abdominal scar left by kidney transplant was experienced positively as it signified a release from the slavery of dialysis and, therefore, hope of getting better. Despite the presence of a bodily mark (of known characteristics and 
dimensions), from the perspective of the informant nurses, this new scar acquired a meaning totally opposite to the scars created by dialysis access.

N5: After a transplant they are much better, they have left behind the dialysis and they have fewer difficulties in accepting this new scar. They have left behind a very hard stage and this scar can acquire a positive meaning. The transplant is positive. Fistulas, on the other hand, always have a negative meaning because they remind them of the treatment. In addition, the fistula is a visible scar that can be felt and that others see and that reminds people of a negative stage. A scar on a kidney is "good". It means that you have got a kidney, that you have stopped dialysis and that you are autonomous and independent and what you can be the one that controls your life.

Despite the positive meaning of kidney transplant scars, continued dialysis access after kidney transplant was perceived negatively. The nurses identified dialysis access as a constant reminder of the feeling of slavery to treatment and of the prior stage.

N1: I think that not having to use a fistula makes the fistula bother you less. To begin with, the simple fact of not inserting needles every other day lightens the load. But it is true that I have found people who, after being transplanted, after a while, they would have liked to have their fistula closed and they have asked about that and they have been advised not to. There are people who do care, especially if it is very large and can cause discomfort. Even if you do not need it, it is still there and can be, a little bit, a constant reminder of what you have been and what you can be again. 


\section{DISCUSSION}

In line with its main objective, this study has identified nurses' perceptions of the effect of CKD and its treatment on individuals' bodies. Of note, the results illustrate the interconnection between the physical and emotional impact of the disease. These observations have been previously explored. Papastavrou et al. (2016) propose that the interpersonal perceptions among nurses and the impact of the disease on the bodies of persons with $\mathrm{CKD}$, as well as the importance of integrating the concept of care with dignity, are essential components of care. At the same time, these authors add that this sensitivity and this specific concept of care cannot be taught but can be learned by working in a context where individuals are treated with dignity and sensitivity and given emotional support. In this way, people with CKD influence nurses' perceptions. There is therefore a transfer of knowledge from patients to nurses through the practice of specific care.

A key finding of this study was that, according to the nurses' perceptions, the bodies of people with CKD were differentiated from those of healthy persons by the dialysis access scar, which was perceived even more negatively if there were complications, such as dysfunction, pain, discomfort, and excessive size. Nurses' perceptions of selfimage and body changes have already been explained in a previous study (Pinto and Siri, 2008), which concluded that, nurses should be aware of the possibility of physical alterations that interfere with self-concept and should be trained to provide an adequate approach.

This finding, presented from the nurses' perspective, is in agreement with those of Kosa, et al., (2016), who reported that people with CKD are most concerned about physical complications (eg, pain, bruising, swelling, bleeding) over and above the interference of dialysis in daily life and infectious complications. The present study also found that the physical scar also had emotional impact, which echoes previous findings in the literature (Silva, et al., 2018), stressing that any physical change in people with CKD requiring dialysis will affect body image. Thus, the perceptions of people with 
CKD reported by Kosa and Silva are replicated by the nurses' perceptions in the present study. In this way, we can assume that nurses empathize with the problems described by people with CKD and support the need to provide adequate answers to the problems identified. It is particularly important to develop person-centered care strategies from a philosophical perspective focused on the humanization of treatments, the provision of individualized services and the absence of infantilization or victimization (Pérez-Rojo, et al., 2018). Returning to the results of the study, these philosophical perspectives should lead to better person-centered care plans that include corporality and the possible effect of treatments on the individual.

In the present study, pain was highlighted as one of the most important effects of complications that can be experienced by people with CKD. Informant nurses defined this pain from a perspective that encompassed all spheres of the person and was not based only on the sensorineural part of "having physical pain". Likewise, the most recent studies analyzing pain in CKD patients describe it as a multidimensional phenomenon with physical, psychological and social components often determined by personal beliefs and cultural values and not only affecting the body. Pain is also viewed in the scientific literature as a phenomenon that transcends neurosensory factors and covers other spheres of the person (Kafkia, et al., 2018).

Another important finding of this study was that people with CKD lost control over their time and experienced the need for dialysis as a form of slavery. The participants linked the treatment with its impact on patients, concluding that it can be perceived as a slavery necessary to survival. Several authors have analyzed the effects of dialysis on individuals' quality of life (Broers, et al., 2018, Paschou, et al., 2018) and have reported similar factors, such as: financial difficulties, loss of independence, tension in personal relationships, significant physiological changes, discomfort or anxiety, loss of previous body image and the desired body image, etc.

Another important issue identified was the lack of understanding of the disease, its treatment and its impact among people with CKD (linked to the above-mentioned feeling of slavery). This lack of understanding was related to the lack of patient information identified by nurses. Here we highlight two important points: the silence of taboo against discussing certain issues and the absence of specific resources needed to improve the situation. To a certain extent, these findings are contradicted by those of other studies (Chan, et al., 2018) reporting that patients acknowledged receiving 
emotional support from their nurses. Possible explanations for this discrepancy are that nurses in this study perceived that support, while not being absent, could be increased, while patients do not expect to receive support and are grateful when it is provided.

Furthermore, in the same line, there is considerable evidence linking a low level of knowledge about the disease itself with higher mortality (Taylor, et al., 2018). In addition, people who were well informed and motivated to participate directly in their care showed greater adherence to the prescribed dietary and drug therapy, thus increasing treatment effectiveness and survival (Umeukeje, et al., 2015, Smith, et al., 2010). In contrast, people with inadequate knowledge, either because of taboo, the difficulty of discussing sensitive issues with professionals, or the lack of professional resources (as found in the present study) are at risk of not understanding or adequately understanding the treatment and, therefore, of poor adherence and a higher risk of mortality. These themes could indicate that the more supported people with CKD feel by the nurses, the better they cope with their illness. This could be related to the fact that the closer the perception nurses have about the disease process, the more humanized the care they provide, approaching the philosophy of person-centered care characterized by empathy and humanity (Leyva-Moral, 2019). These practices, centered on emotional care and educational reinforcement can be strongly affected by issues such as high nursing workloads and the nurse-patient ratio (Papastavrou, Efstathiou \& Andreou, 2016), which were also found in the discourse analyzed. As described, these characteristics affect nurses' resources and, therefore, also affect their professional work and the care provided.

Some authors have attempted to analyze people's beliefs about their disease and its treatment. Several studies have concluded that people's perceptions of their disease can be modified through specialized psychological intervention and educational training directly involving the patient in these processes. These studies also report that these strategies can be more effective if they are carried out before the beliefs and perceptions have become entrenched (Clarke, et al., 2016; Jansen, et al., 2013). Therefore, specific interventions by nurses could help to influence people's perceptions of their disease and its treatment and thus improve their clinical and psychological outcomes. Nevertheless, the strength of beliefs about disease vary and consequently require different strategies. The results of this study and current scientific evidence suggest that the support of 
specialized professionals with specific training in the impact of disease on body image is essential.

Analysis of the results of this study show that, unlike dialysis access scars, kidney transplant scars were viewed as positive, signifying an end to the slavery of dialysis. This new phase was defined by the interviewees as being characterized by hope because of the benefits of transplantation on recipients' quality of life. Of note, the literature clearly describes that this phase also involves obvious negative effects on the renal transplant recipient's body, such as weight gain, the appearance of body hair, and the abdominal scar itself (Zimbrean, 2015). Nevertheless, the discourse analyzed in this study revealed a general perception that transplant scars were associated with a considerable enhancement in people's quality of life and a more hopeful attitude to the illness, its treatment and body image.

In contrast, the continuing existence of a functioning dialysis access after transplantation served as a reminder of slavery to CKD and its treatment. Several studies have examined the possibility of removing dialysis access after a kidney transplant, but have concluded that it is not the best option because dialysis access remains viable for a certain time and having a functioning dialysis access can facilitate the return to the former treatment method (Voorzaat and Rotmans, 2017). The psychological impact of continuing to have a functioning dialysis access after a kidney transplant has also been explored, and the recommendations were the same, as there were no major psychological repercussions of continued access (Yaffe and Greenstein, 2012). Thus, these studies recommend patient education to convey the importance of the continuing care of dialysis access, although it may not be needed for a certain period of time. This gives rise to the constant memory of the previous phase identified by nurses participating in this study and to the possibility of a return to this phase in the future.

The above findings explain why the participants in this study detected a close connection between the physical and emotional impact of CKD. After years of experience, the interviewees described this connection between psyche and soma and critically reflected on their performance as professionals, reaching important and farreaching conclusions. 


\section{LIMITATIONS OF THE STUDY}

The main limitation of the study is the small sample size. As previously mentioned, data saturation, defined by the non-appearance of new themes in the discourse analyzed, was achieved with 6 informants. Our explanation is the closeness among informants, leading to a highly specialized and interconnected team. Despite this limitation, this study applied all the criteria for rigor pertaining to the field of qualitative research. Future research on body image in CKD is needed to deepen the findings of this study, given that the issue is an important and overlooked topic that may have a profound impact on people with CKD and their care.

Future studies could consider applying the knowledge garnered in this study, focusing on each of the aspects identified, such as: meanings attributed to scars, barriers to providing correct information and patient education on their illness and treatments, nursing thinking as an agent of reflection, and nurses' perceptions of the processes experienced by others, etc.

\section{IMPLICATIONS FOR PRACTICE}

The close connection found between the physical and the emotional in this study, as well as the taboo about speaking about intimate issues and the lack of resources, suggests the need for psychological training among nurses as well as greater emphasis on patient education. These findings could influence daily practice and strategies to improve the nursing profession. Future research is needed to establish and deepen these bases. At the same time, proposals for improvement should be considered by management with the aim of enhancing care standards. 


\section{CONCLUSIONS}

Bodily changes occurring after diagnosis of CKD have an emotional impact on individuals' well-being. These findings have been reported in the voice of the nurses who empathize with the problems described by people with CKD and support the need to provide adequate answers. It is particularly important to develop person-centered care strategies and ensure that nurses obtain the necessary tools for the development of their profession and activities, thus overcoming the barriers analyzed. At the same time, nurses should feel supported by a multidisciplinary team composed of all professionals acting in this sphere.

Some of the nurses' perceptions identified in this study contradict scientific knowledge. According to the informants, the scar of the kidney transplant may represent hope to their patients and have positive connotations. Thus, the changes occurring in the bodies of persons with $\mathrm{CKD}$ can transcend the physical sphere and acquire more complex meanings. These meanings are elucidated by nurses' thinking, including all the domains of the person and providing important meanings for an understanding of daily practice. In turn, this knowledge acquisition among nurses is strongly influenced by their interpretation of their patients' experiences. 


\section{REFERENCES}

Broers N.J.H., Martens R.J.H., Canaud B., et al. (2018). Health-related quality of life in end-stage renal disease patients: the effects of starting dialysis in the first year after the transition period. Int Urol Nephrol, 50(6), 1131-1142.

Chan Z.C., Fung Y. \& Chien W. (2013). Bracketing in phenomenology: Only undertaken in the data collection and analysis process. The Qualitative Report, 18(30), 1-9.

Chan E.A., Wong F., Cheung M.Y. et al. (2018). Patients' perceptions of their experiences with nurse-patient communication in oncology settings: A focused ethnographic study. PLoS ONE, 13(6), e0199183.

Clarke A.L., Yates T., Smith A.C. et al. (2016). Patient's perceptions of chronic kidney disease and their association with psychosocial and clinical outcomes: a narrative review. Clinical Kidney Journal, 9(3), 494-502

Colaizzi P.F. (1978). Psychological research as the phenomenologist views. In: Sanders C. (2003). Application of Colaizzi's method: Interpretation of an auditable decision trail by a novice researcher. Contemporary Nurse, 14, 292-302.

Gordon M. (1994). Nursing Diagnosis: Process and application. (3rd ed.). St. Louis: Mosby.

Guba E.G. (1981). Criteria for Assessing the Trustworthiness of Naturalistic Inquiries. ERIC/ECTJ, 29(2), 75-91.

Heidegger M. (2010). The phenomenology of religious life. (1st ed.). United States of America: Indiana University Press.

Jansen D.L., Heijmans M.J.W.M., Rijken M. et al. (2013). Illness perceptions and treatment perceptions of patients with chronic kidney disease: Different phases, different perceptions? British Journal of Health Psychology, 18, 244-262

Kafkia T., Vehvilainen-Julkunen K., Zyga S. et al. (2018). Chronic Kidney Disease and Pain Perception. International Journal of Caring Sciences, 11(1), 580-589.

Kosa S.D., Bhola C. \& Lok C.E. (2016). Hemodialysis patients' satisfaction and perspectives on complications associated with vascular access related interventions: are we listening? J Vasc Access, 17(4), 313-319.

Kuper A., Lingard L. \& Levinson W. (2008). Critically appraising qualitative research. BMJ, 337, 687-692. 
Lewis H. \& Arber S. (2015). The role of the body in end-stage kidney disease in young adults: Gender, peer and intimate relationships. Chronic Illn, 11(3), 184-97.

Leyva-Moral J.M. (2019). Are we ready to provide real humanized care? Rev Exp Med, 5(1), 3-4.

Malheiro-Oliveira P. \& Arruda-Soares D. (2012). Percepciones de las personas con insuficiencia renal crónica sobre la calidad de vida. Enfermería Global, 28, 257-275.

Martínez-Castelao A., Troya M., Cases A. et al. (2017). Registre de malalts renals de Catalunya. (1ra ed.). Barcelona: ServeiCatalà de la Salut.

Ormandy P. (2008). Information topics important to chronic kidney disease patients: a systematic review. Journal of Renal Care, 34(1), 19-27.

Papastavrou E., Efstathiou G. and Andreou C. (2016). Nursing students' perceptions of patient dignity. Nursing Ethics, 23(1), 92-103.

Partridge K.A. \& Robertson N. (2011). Body-image disturbance in adult dialysis patients. Disability and Rehabilitation, 33(6), 504-510.

Paschou A., Damigos D., Skapinakis P., et al. (2018). The Relationship between Burden and Depression in Spouses of Chronic Kidney Disease Patients. Depress Res Treat, 13, 8694168.

Pendse S.S., Singh, A. \& Zawada Jr, E. (2006). Initiation of dialysis. In: J.T. Daugirdas P.G. \& Blake T.S. Handbook of dialysis (4th ed.) (pp. 14-21). Philadelphia: Lippincott Williams \& Wilkins.

Pérez-Rojo G., Noriega C., Velasco C., et al. (2018). Development and assessment of the content validity of the professional good practices scale in nursing homes. Int Psychogeriatr, 7, 1-5.

Pinto K.K.O. \& Spiri W.C. (2008). Nurses' perception concerning the care for patients with physical disabilities that interfere with self-image: a phenomenological approach. Revista Latino-Americana de Enfermagem, 16(3), 407-413.

Roy C., Whetsell M.V. \& Frederickson K. (2009) The Roy adaptation model and research. NursSci Q, 22(3), 209-211.

Sadeghian J., Masroor-Roudsari D., Seyedfatemi N. et al. (2016). Body Image Disturbance: A Comparative Study among Haemodialysis and Kidney Transplant Patients. Journal of Clinical and Diagnostic Research for doctors, 10(5), 14-16.

Silva D.M.D., Silva R.M.C.R.A., Pereira E.R., et al. (2018). The body marked by the arteriovenous fistula: a phenomenological point of view. Rev Bras Enferm, 71(6), 2869-2875.

Smith K., Coston M., Glock K. et al. (2010). Patient Perspectives on Fluid Management in Chronic Hemodialysis. J Ren Nutr, 20(5), 334-341.

Taylor D.M., Fraser S., Dudley C., et al. (2018) Health literacy and patient outcomes in chronic kidney disease: a systematic review. Nephrol Dial Transplant, 33(9), 15451558 .

Umeukeje E.M., Merighi J.R., Browne T. et al. (2015). Self-Motivation Is Associated with Phosphorus Control in End-Stage Renal Disease. J Ren Nutr, 25(5), 433-439. 
Vargas-Marcos F. (2015). Documento Marco sobre Enfermedad Renal Crónica (ERC) dentro de la Estrategia de Abordaje a la Cronicidad en el SNS. España: Misisterio de Sanidad, Servicios Sociales e Igualdad.

Voorzaat B.M., Rotmans J. (2017). Authors' reply to Comment on: The pros and cons of preserving a functioning arteriovenous fistula after kidney transplantation. $J$ Vasc Access, 18(4), e55.

Yaffe H.C. \& Greenstein, S.M. (2012). Should functioning AV fistulas be ligated after renal transplantation? J Vasc Access, 13(4), 405-408.

Zalai D., Szeifert L. \& Novak, M. (2012). Psychological Distress and Depression in Patients with Chronic Kidney Disease. Seminars in Dialysis, 25(4), 428-438.

Zimbrean P.C. (2015). Body image in transplant recipients and living organ donors. Curr Opin Organ Transplant, 20(2), 198-210.

\section{TABLES AND FIGURES}

TABLE 1. PARTICIPANTS' DATA

\begin{tabular}{cclcc}
\hline Participant & Age & Gender & Experience & Shift \\
\hline Nurse 1 (N1) & 36 years & Female & 16 years & Late Shift \\
Nurse 2 (N2) & 51 years & Female & 24 years & Early Shift \\
Nurse 3 (N3) & 62 years & Female & 28 years & Early Shift \\
Nurse 4 (N4) & 41 years & Female & 16 years & Early Shift \\
Nurse 5 (N5) & 31 years & Female & 11 years & Late Shift \\
Nurse 6 (N6) & 31 years & Female & 11 years & Late Shift \\
Interviewer & 29 years & Male & 8 years & Late Shift \\
\hline
\end{tabular}


TABLE 2. THEMES AND SUBTHEMES

Themes

From physical pain to emotional pain in the patient's body

\section{Subthemes}

Stigma created by the need for dialysis

Dysfunctionality accompanied by pain

The feeling of slavery

Lack of patient information

The silence of taboo

Lack of resources

Hope for improvement 


\section{APPENDIX}

\section{APPENDIX 1. INTERVIEW GUIDE}

Date:

Place of the interview:

Age:

Gender:

Time as a nephrology nurse:

1. What is your perception of the changes that occur in the body of the renal patient throughout the disease process?

2. Comment on the importance of the nursing interventions performed on this patient in relation to the impact on their bodies.

3. What key actions are derived from the nurse care in the process of accompanying the patients (in relation to the CKD body impact)?

4. Do you think there are any strategies for change or proposals for improvement in the nephrological patient care process (in relation to the CKD body impact)? 